\begin{tabular}{|c|l|}
\hline Title & Synthesis of monodispersed silica nanoparticles with high concentration by the Stober process \\
\hline Author(s) & Tadanaga, Kiyoharu; Morita, Koji; Mori, Keisuke; Tatsumi sago, Masahiro \\
\hline Citation & $\begin{array}{l}\text { Journal of sol-gel science and technology, 68(2), 341-345 } \\
\text { https://doi.org/10.1007/\$10971-013-3175-6 }\end{array}$ \\
\hline Issue Date & 2013 11 \\
\hline Doc URL & http://hdl.handle.net/2115/57359 \\
\hline Type & article (author version) \\
\hline File Information & silica particles paper final version 140305.pdf \\
\hline
\end{tabular}

Instructions for use 


\title{
Synthesis of Monodispersed Silica Nanoparticles with High Concentration by the
}

\section{Stöber Process}

\author{
*Kiyoharu Tadanaga ${ }^{1}$, Koji Morita $^{1,2}$, Keisuke Mori $^{1}$, and Masahiro Tatsumisago ${ }^{1}$ \\ ${ }^{1}$ Department of Applied Chemistry, Graduate School of Engineering, \\ Osaka Prefecture University, 1-1 Gakuen-cho, Naka-ku, Sakai, Osaka 599-8531, Japan \\ ${ }^{2}$ Nippon Bee Chemical Co., Ltd. \\ 2-14-1 Shodai-Ohtani Hirakata, Osaka 573-1153, Japan
}

\begin{abstract}
Silica nanoparticles with high concentration were prepared by the sol-gel process based on the Stöber method using tetraethoxysilane (TEOS) as a starting material. It was found that silica sol with about $4 \mathrm{wt} \%$ in concentration and with a diameter of about 10 nm was obtained by controlling the reaction conditions in the Stöber process. By removing the solvent under a reduced pressure, the particle concentration was increased up to $15 \mathrm{wt} \%$ without aggregation.
\end{abstract}

Keywords : the Stöber method, monodispersed particles, silica

*Corresponding author

(present address)

Kiyoharu Tadanaga

Faculty of Engineering, Hokkaido University

E-mail: tadanaga@eng.hokudai.ac.jp

Tel \& Fax +81-11-706-6572 


\section{Introduction}

Preparation of mono-disperse inorganic nanoparticles has attracted a considerable attention because of their potential importance in technological applications in the fields of optical devises, catalysts, filler for polymers, and so on. Among synthesis techniques of particles, a liquid phase process, especially sol-gel process, is a superior one to prepare monodispersed particles [1-5]. The so-called "Stöber method" is an excellent process to prepare monodispersed silica particles using sol-gel process [6], and silica particles with a diameter of about $100 \mathrm{~nm}$ to a few $\mu \mathrm{m}$ are easily prepared by this method [7].

Much smaller silica particles are required for some applications. For example, in a hard-coating for polymer substrates, the coating layer should be transparent, and thus the silica particles added to the coating must be smaller than a few tens of nanometer to avoid scattering. However, the Stöber method is believed that the preparation of uniform-sized silica nanospheres with a size of below $50 \mathrm{~nm}$ is rather difficult $[8,9]$. To obtain much smaller nano-particles, e.g., about $10 \mathrm{~nm}$ in diameter, preparation of silica nanoparticles by modified Stöber method using basic amino acid such as L-lysine $[8,9]$ or by water in oil micro-emulsion process $[10,11]$ have been reported. In the industrial scale, colloidal silica with small diameter has been prepared using water glass 
[12].

Those silica nanoparticles are usually prepared under highly diluted conditions. However, for the use of those nanoparticles to the hard-coating for the polymer substrates, high concentration of silica is preferred. In addition, the surface of the commercial particles is covered with surfactants or dispersing agents. About the surface of nanoparticles, the surface must be modified with some silane-coupling agents to form covalent bonding with matrix of the hard-coatings, and thus, the nanoparticles without surfactants or dispersing agent are preferable. There are plenty of studies on the preparation of silica nanoparticles by the Stöber method. However, not so many studies are reported on the preparation of high-concentration of silica nanoparticles without using surfactants or dispersing agent.

In this study, monodispersed silica nanoparticles with a diameter of less than $20 \mathrm{~nm}$ and with high $\mathrm{SiO}_{2}$ concentration (up to $15 \mathrm{wt} \%$ ) were prepared by the sol-gel process based on the Stöber method, by the control of the preparation conditions. Effects of composition of starting materials on the size and distribution of silica particles were examined. In addition, the solvent in the prepared sol was removed under a reduced pressure to increase the concentration of silica. Effects of concentration process for the size and distribution of silica nanoparticles were examined. 


\section{Experimental}

2.1. Preparation of silica nano-particles

Silica particles were prepared through a process based on the Stöber method, using tetraethoxysilane (TEOS) (Shin-Etsu Chemical, Japan) as a starting material. TEOS was dissolved in ethanol (Wako Pure Chemical Industries, Japan). Separately, ammonia water $(25 \mathrm{wt} \%)$ and ethanol were mixed. Then, two solutions were mixed, and then the obtained sol was stirred for $24 \mathrm{~h}$. The particles in the sols were collected by centrifugation and dried in vacuo for $12 \mathrm{~h}$. The mole ratios of TEOS : $\mathrm{H}_{2} \mathrm{O}(25$ wt $\left.\% \mathrm{NH}_{4} \mathrm{OHaq}\right)$ : ethanol were (a) $\mathrm{x}: 126: 9(1<\mathrm{x}<15)$, (b) $5: \mathrm{y}: 9(63<\mathrm{y}<126)$, and (c) $5: 79: \mathrm{z}(9<\mathrm{z}<14)$.

To increase the concentration of silica, the solvent in the prepared sol was removed under a reduced pressure using a rotatory evaporator at $55^{\circ} \mathrm{C}$.

The concentration of $\mathrm{SiO}_{2}$ in the sol was determined from the weight of the collected and heat-treated (at $1000^{\circ} \mathrm{C}$ ) particles versus the total weight of the prepared sol. The yield of the $\mathrm{SiO}_{2}$ was calculated from the weight of the collected and heat-treated (at $1000^{\circ} \mathrm{C}$ ) particles versus the theoretical weight of $\mathrm{SiO}_{2}$ obtained from TEOS. 


\subsection{Materials characterization}

A transmission electron microscope (TEM, JEOL JEM2010) was used for the observation of shape and size of the particles. Size distribution of particles in the sol was determined using a dynamic light scattering (DLS6000, Otsuka denshi). Thermal properties of the obtained particles were determined using DTA-TG (Themo Plus TG8120, Rigaku), with a heating rate of $10^{\circ} \mathrm{C} / \mathrm{min}$.

\section{Results and discussion}

\subsection{Preparation of $\mathrm{SiO}_{2}$ particles with various molar ratios in the starting materials}

Silica nanoparticles were prepared using the Stöber process and according to the reference [11], where the molar ratio of TEOS : EtOH : $\mathrm{H}_{2} \mathrm{O}\left(25 \mathrm{wt} \% \mathrm{NH}_{4} \mathrm{OHaq}\right)$ is 1 : 126 : 9. Figure 1 shows a TEM image of the nanoparticles obtained, and size distribution of the nanoparticles determined by dynamic light scattering (DLS). From the TEM image, the diameter of the primary particles is about $10 \mathrm{~nm}$. The average particle diameter in the sol and geometric standard deviation, determined by the DLS, were $8.5 \mathrm{~nm}$ and 1.22 , respectively. These results are in good agreement with the TEM observation, and indicate the rather good monodispersity of the particles. In this 
composition, the $\mathrm{SiO}_{2}$ concentration in the sol is less than $1 \%$. Under such a dilute condition, silica nanoparticles with a diameter of about $10 \mathrm{~nm}$ were obtained.

To increase the $\mathrm{SiO}_{2}$ concentration in the sol, the molar ratio of TEOS was increased, according to the molar ratio series (a), where TEOS : EtOH : $\mathrm{H}_{2} \mathrm{O}(25 \mathrm{wt} \%$ $\left.\mathrm{NH}_{4} \mathrm{OHaq}\right)$ was $\mathrm{x}: 126: 9(1 \leq \mathrm{x} \leq 15)$. Figure 2 shows (a) average particle size and geometric standard deviation and (b) particle concentration and yield, with changing TEOS ration in the starting materials. With an increase in $\mathrm{x}$ (TEOS ratio) up to 10, the concentration of $\mathrm{SiO}_{2}$ is increased, but the average particle size is less than $10 \mathrm{~nm}$. Concentration of $\mathrm{SiO}_{2}$ was about 3 wt\% with $\mathrm{x}=10$. When the $\mathrm{x}$ was larger than 10 , the water for the hydrolysis of TEOS was not enough. Thus, unreacted TEOS may remain in the sol, and the concentration of $\mathrm{SiO}_{2}$ was leveling off with larger TEOS content.

To increase the $\mathrm{SiO}_{2}$ concentration in the sol, the molar ratio of EtOH was decreased, according to the molar ratio series (b), where TEOS : EtOH : $\mathrm{H}_{2} \mathrm{O}(25 \mathrm{wt} \%$ $\left.\mathrm{NH}_{4} \mathrm{OHaq}\right)$ was $5: \mathrm{y}: 9(63 \leq \mathrm{y} \leq 126)$. Figure 3 shows (a) the average particle size and geometric standard deviation and (b) particle concentration and yield, with changing $\mathrm{EtOH}$ ratio in the starting materials. With a decrease in $\mathrm{y}$ ( $\mathrm{EtOH}$ ratio), the concentration of the obtained $\mathrm{SiO}_{2}$ is increased, but the average particle size is increased. 
With the smallest $y$ in the series (b) $(y=63$, largest TEOS concentration), the concentration of the obtained $\mathrm{SiO}_{2}$ was about $5 \%$ and the average particle size is about $18 \mathrm{~nm}$.

To promote the hydrolysis and condensation reaction of TEOS, which should result in the increase in the $\mathrm{SiO}_{2}$ concentration, the $\mathrm{H}_{2} \mathrm{O}$ molar ratio was increased. The sol was prepared in the molar ratio series (c), where TEOS : EtOH : $\mathrm{H}_{2} \mathrm{O}(25 \mathrm{wt} \%$ $\left.\mathrm{NH}_{4} \mathrm{OHaq}\right)$ was $5: 79: \mathrm{z}(9 \leq \mathrm{z} \leq 14)$. Figure 4 shows (a) average particle size and geometric standard deviation and (b) particle concentration and yield, with changing $\mathrm{H}_{2} \mathrm{O}\left(25 \mathrm{wt} \% \mathrm{NH}_{4} \mathrm{OHaq}\right)$ ratio in the starting materials. With an increase in $\mathrm{z}\left(\mathrm{H}_{2} \mathrm{O}\right.$ ratio), the concentration of the obtained $\mathrm{SiO}_{2}$ is increased, but the average particle size is largely increased up to about $60 \mathrm{~nm}$.

\subsection{Increase of the $\mathrm{SiO}_{2}$ concentration by removal of solvent from the obtained sol}

To increase the concentration of silica, the solvent in the prepared sol was removed under a reduced pressure using a rotatory evaporator at $55^{\circ} \mathrm{C}$. In this experiment, the sol prepared with TEOS : EtOH $: \mathrm{H}_{2} \mathrm{O}\left(25 \mathrm{wt} \% \mathrm{NH}_{4} \mathrm{OHaq}\right)=5: 79: 9$ was used, and initial $\mathrm{SiO}_{2}$ concentration was about $4 \%$. By controlling the heating time, the sols with $\mathrm{SiO}_{2}$ concentration of $7,11,15$, and 31 were obtained. The Particle concentration and size of 
silica nanoparticles are shown in Table 1. In the condensed sol with $\mathrm{SiO}_{2}$ concentration of 7,11 , and 15 , the average particle size determined by DLS is less than $10 \mathrm{~nm}$, and the sol was almost transparent. In addition, the sol was confirmed to be stable for a few weeks. These results suggest that the particles are not aggregated during concentration of the sol up to $15 \mathrm{wt} \%$. The $\mathrm{pH}$ of the sol was changed from $\mathrm{pH}$ of 11 to $\mathrm{pH}$ of 7 with the removal of solvent, because $\mathrm{NH}_{3}$ is also removed with the solvent. This change of $\mathrm{pH}$ (elimination of $\mathrm{NH}_{3}$ from the sol) must contribute to the stability of the concentrated sol. However, when the sol was concentrated to $30 \mathrm{wt} \%$, the particle size is more than $10 \mathrm{~nm}$, and the sol became translucent. This suggests that the particles are highly aggregated during concentration of the sol. Figure 5 shows the FE-SEM image of silica nanoparticles (a) before and (b) after the concentration to 15 $\mathrm{wt} \% \mathrm{SiO}_{2}$. The size of the primary particle is almost the same before and after the concentration, and is less than about $20 \mathrm{~nm}$. DLS results supported the FE-SEM observation, where the average particle size was about $10 \mathrm{~nm}$ and the geometric standard deviation was 1.2. The aggregated particles observed in the FE-SEM images must be formed during centrifugation and drying for the FE-SEM observation.

It is found that silica sol with $4 \mathrm{wt} \% \mathrm{SiO}_{2}$ concentration and with an average diameter of less than $10 \mathrm{~nm}$ can be obtained by the control of chemical composition in 
the starting materials. By the combination of chemical composition control and solvent evaporation, silica sol with $\mathrm{SiO}_{2}$ concentration of $15 \mathrm{wt} \%$ and with an average particle size of less than $10 \mathrm{~nm}$. In this process, surfactant or dispersing agents were not used. Thus, the surface of $\mathrm{SiO}_{2}$ particles in the sol must be easily modified with a silane-coupling agent. In addition, the sol with such high concentration and with small particle size is very attractive for the use of filler for the hard-coating.

\section{Conclusions}

Silica nanoparticles with high concentration were prepared by the Stöber method using TEOS as a starting material. It was found that about $4 \mathrm{wt} \%$ of silica nanoparticles with a diameter of about $10 \mathrm{~nm}$ were obtained by controlling the reaction conditions in the Stöber process. By removing solvent under a reduced pressure, the particle concentration was increased up to $15 \mathrm{wt} \%$ without aggregation. 


\section{Reference}

[1] Gellermann C, Storch W, Wolter H (1997) Synthesis and characterization of the organic surface modifications of monodisperse colloidal silica. J. Sol-Gel Sci. Techol., 8 173-176.

[2] Van Cantfort O, Michaux B, Pirard R, Pirard JP, Lecloux AJ (1997) Synthesis and characterization of monodisperse spherical zirconia particles. J. Sol-Gel Sci. Technol., 8, 207-211.

[3] Mine E, Hirose M, Kubo M, Kobayashi Y, Nagao D, Konno M (2006) Synthesis of submicron-sized titania-coated silica particles with a sol-gel method and their application to colloidal photonic crystals. J. Sol-Gel Sci. Technol., 38, 91-95.

[4] Kim KD, Kim HT(2002) Formation of silica nanoparticles by hydrolysis of TEOS using a mixed semi-batch/batch method. J. Sol-Gel Sci. Technol., 25 183-189.

[5] Ishida H, Tadanaga K, Hayashi A, Tatsumisago M (2013) Synthesis of monodispersed lithium silicate particles using the sol-gel method. J. Sol-Gel Sci. Technol., 65, 41-45.

[6] Stöber W, Fink A, Bohn E (1968) Controlled growth of monodisperse silica spheres in micron size range. J. Colloid Interface Sci., 26, 62-69.

[7] Nishimori H, Tatsumisago M, Minami T (1997) Growth Mechanism of Large 
Monodispersed Silica Particles Prepared from Tetraethoxysilane in the Presence of Sodium Dodecyl Sulfate. J. Sol-Gel Sci. Technol., 9, 25-31.

[8] Yokoi T, Sakamoto Y, Terasaki O, Kubota Y, Okubo T and Tatsumi T (2006) Periodic arrangement of silica nanospheres assisted by amino acids. J. Am. Chem. Soc., 128, 13664-13665.

[9] Yokoi T, Wakabayashi J, Otsuka Y, Fan W, Iwama M, Watanabe R, Aramaki K, Shimojima A, Tatsumi T, Okubo T (2009) Mechanism of Formation of Uniform-Sized Silica Nanospheres Catalyzed by Basic Amino Acids. Chem. Mater., 21, 3719-3729. [10] Arriagada FJ, Osseo-Asare K (1999) Synthesis of nanosize silica in a nonionic water-in-oil microemulsion: Effects of the water/surfactant molar ratio and ammonia concentration. J. Colloid Interface Sci., 221, 210-220.

[11] Naka Y, Komori Y, Yoshitake H (2010) One-pot synthesis of organo-functionalized monodisperse silica particles in W/O microemulsion and the effect of functional groups on addition into polystyrene. Colloids Surf. A, 361, 162-168.

[12] Masuda N, Ota S (2008) Silica sol and process for production thereof. World Patent WO/2008/015943. 


\section{Figure captions}

Figure 1 (a) TEM image of silica nanoparticles and (b) Size distribution of silica nanoparticles determined by DLS. The molar ratio of TEOS : EtOH : $\mathrm{H}_{2} \mathrm{O}(25 \mathrm{wt} \%$ $\left.\mathrm{NH}_{4} \mathrm{OHaq}\right)$ is $1: 126: 9$.

Figure 2 (a) Average particle size and geometric standard deviation and (b) particle concentration and yield, with changing TEOS ration in the starting materials. The molar ratio of TEOS : EtOH : $\mathrm{H}_{2} \mathrm{O}\left(25 \mathrm{wt} \% \mathrm{NH}_{4} \mathrm{OHaq}\right)$ is $\mathrm{x}: 126: 9(1 \leq \mathrm{x} \leq 15)$.

Figure 3 (a) Average particle size and geometric standard deviation and (b) particle concentration and yield, with changing EtOH ratio in the starting materials. The molar ratio of TEOS : $\mathrm{EtOH}: \mathrm{H}_{2} \mathrm{O}\left(25 \mathrm{wt} \% \mathrm{NH}_{4} \mathrm{OHaq}\right)$ is $5: \mathrm{y}: 9(63 \leq \mathrm{y} \leq 126)$

Figure 4 (a) Average particle size and geometric standard deviation and (b) particle concentration and yield, with changing $\mathrm{H}_{2} \mathrm{O}$ (25 wt\% $\mathrm{NH}_{4} \mathrm{OHaq}$ ) ratio in the starting materials. The molar ratio of TEOS : EtOH : $\mathrm{H}_{2} \mathrm{O}\left(25 \mathrm{wt} \% \mathrm{NH}_{4} \mathrm{OHaq}\right)$ is $5: 79: \mathrm{z}(9 \leq$ $\mathrm{z} \leq 14)$

Figure 5 FE-SEM images of silica nanoparticles (a) before and (b) after the concentration to $15 \mathrm{wt} \% \mathrm{SiO}_{2}$. 
Table 1 Particle concentration and size of silica nanoparticles in concentration process

\begin{tabular}{|l|ccccc|}
\hline & $\begin{array}{c}\text { Original } \\
\text { sol }\end{array}$ & Step1 & Step 2 & Step 3 & Step 4 \\
\hline $\mathrm{SiO}_{2}$ concentration / wt\% & 3.9 & 7.0 & 11.2 & 15.3 & 31.2 \\
Average diameter / nm & 7.6 & 4.4 & 4.0 & 4.5 & 12.0 \\
\hline
\end{tabular}




\section{$20 \mathrm{~nm}$}

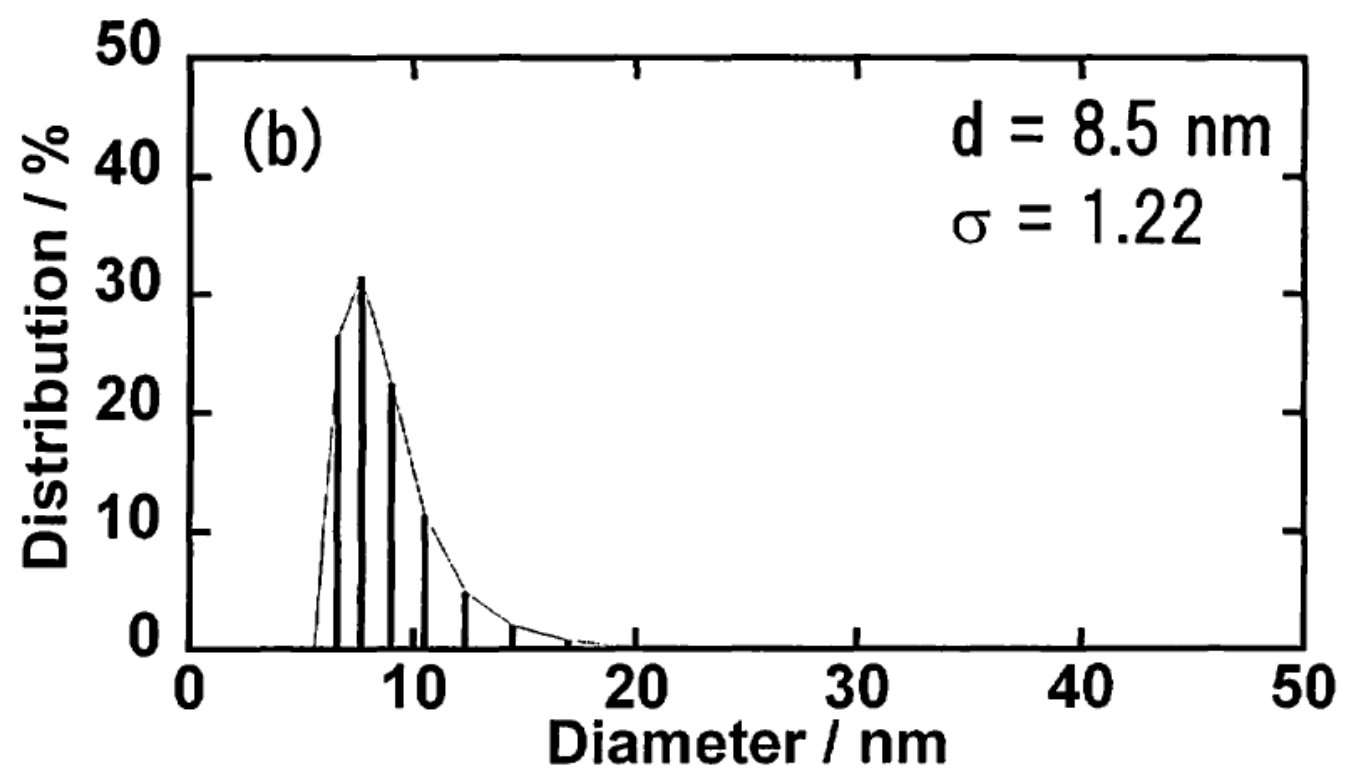

Figure 1 

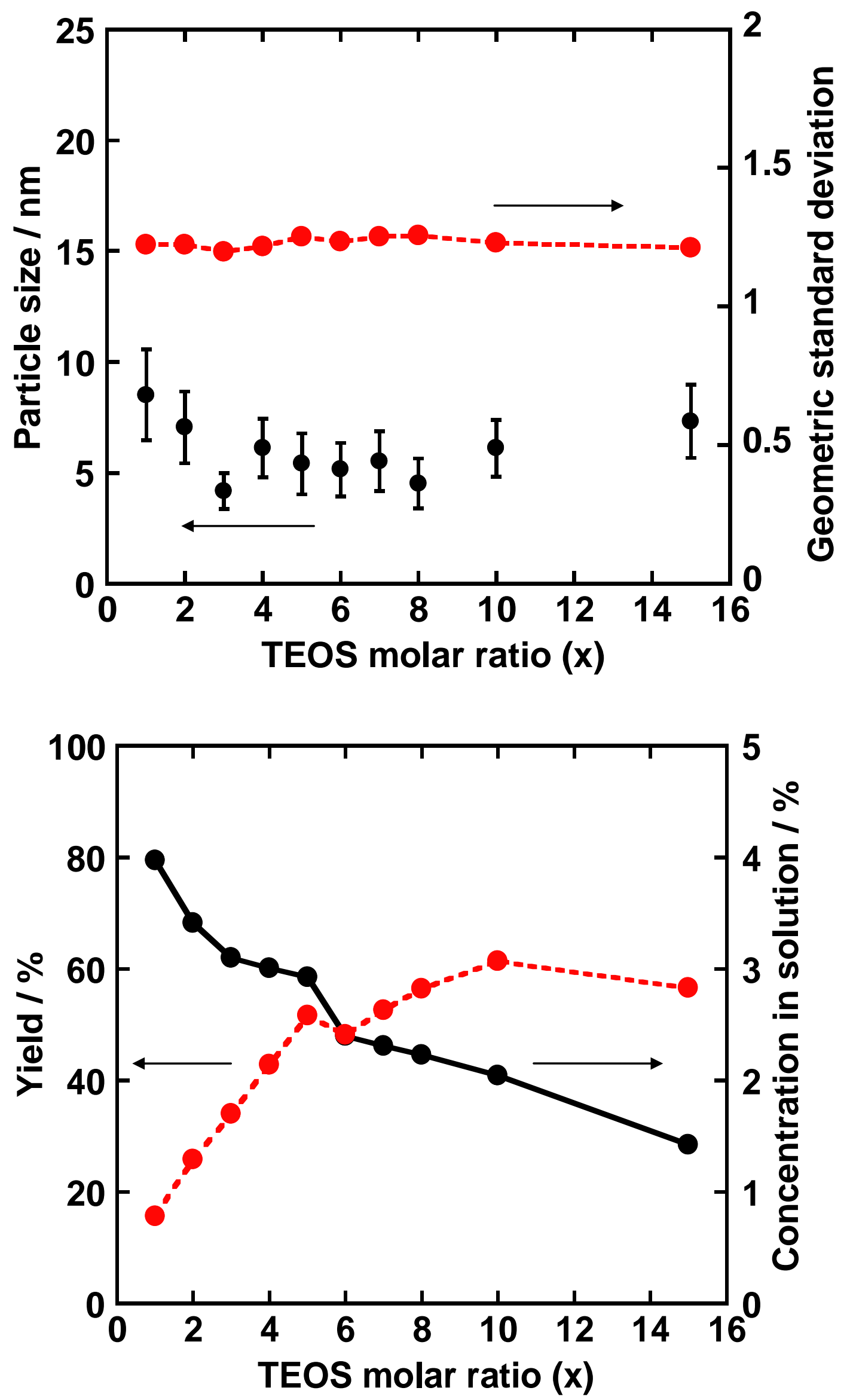

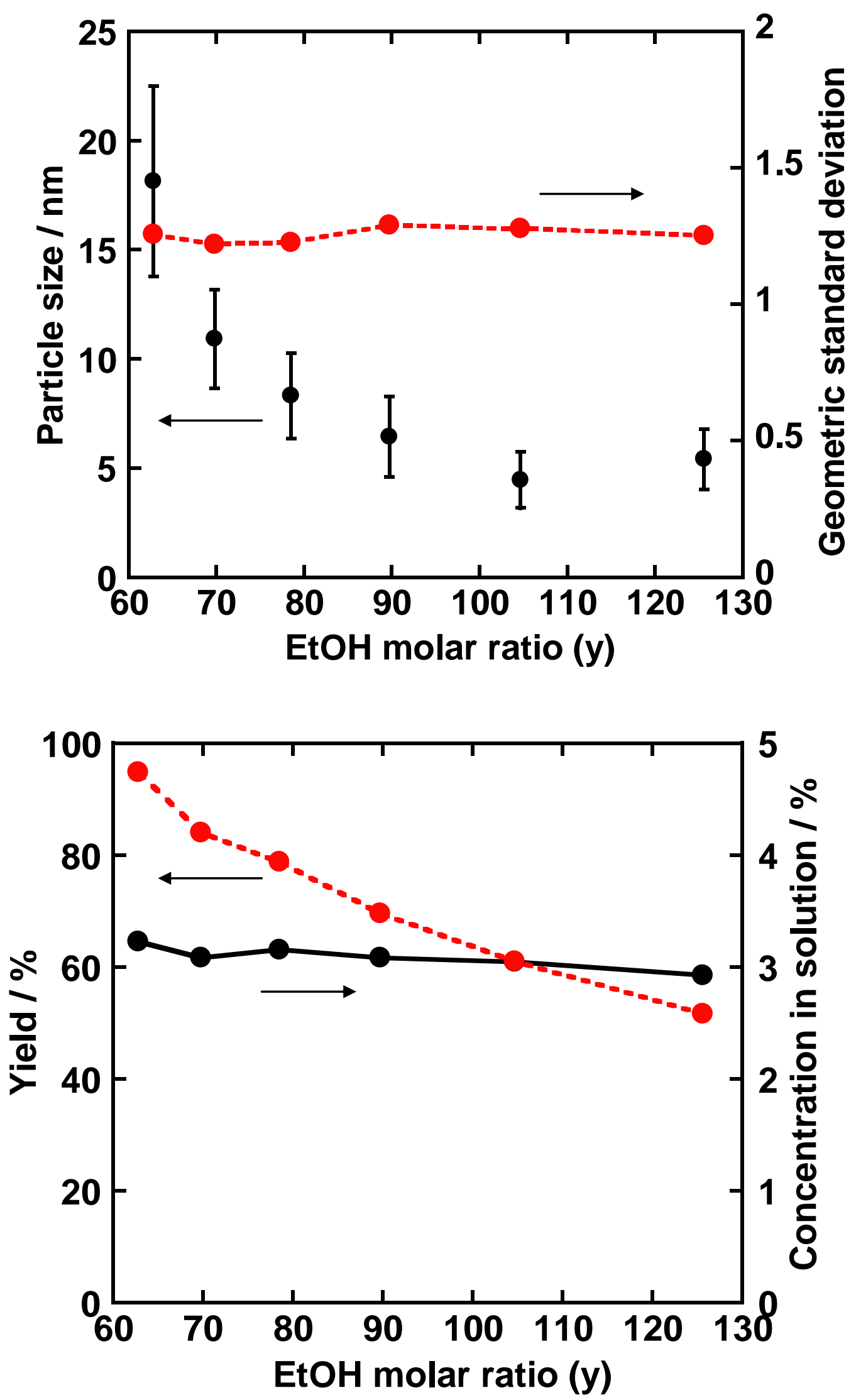

Figure 3 

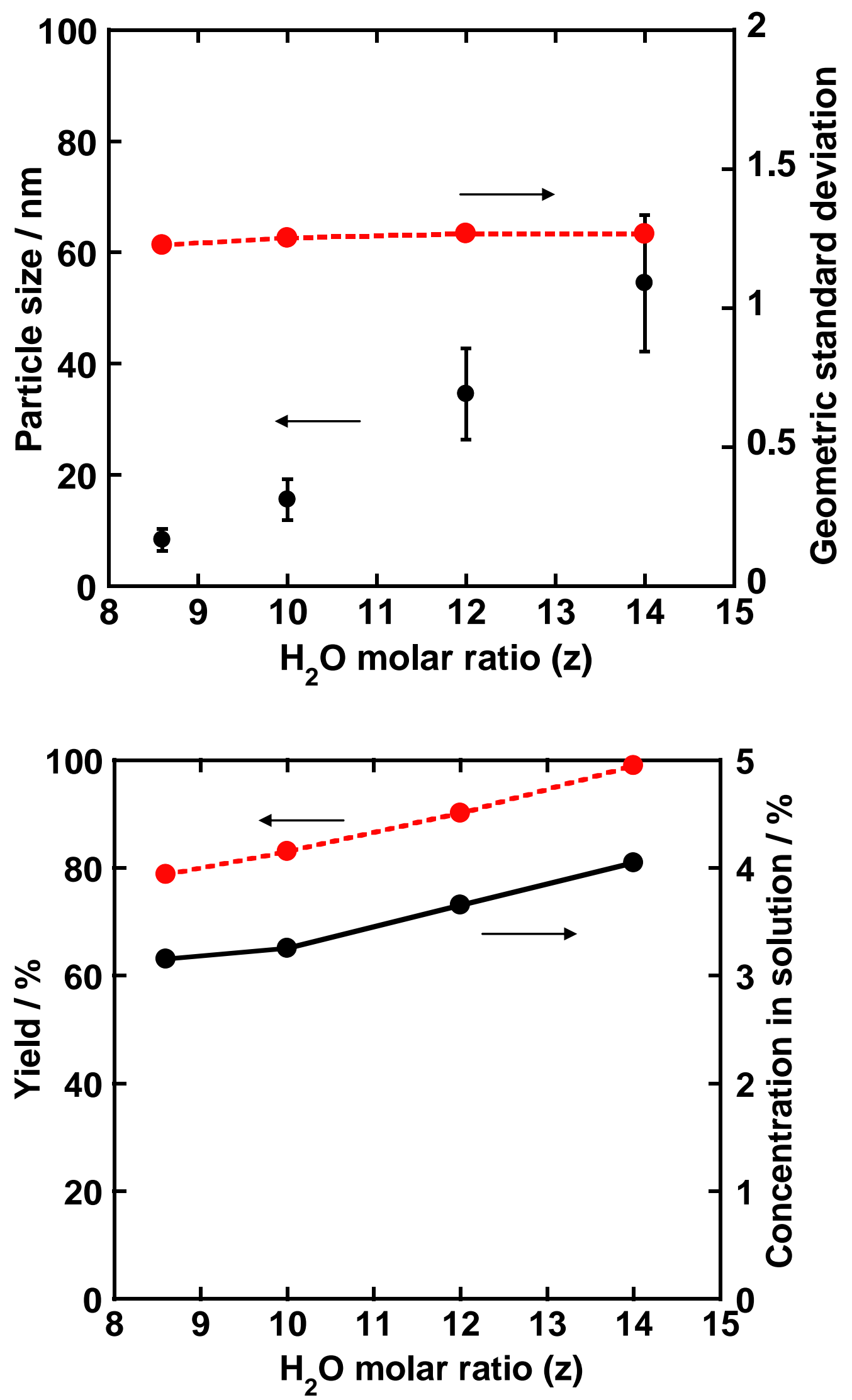

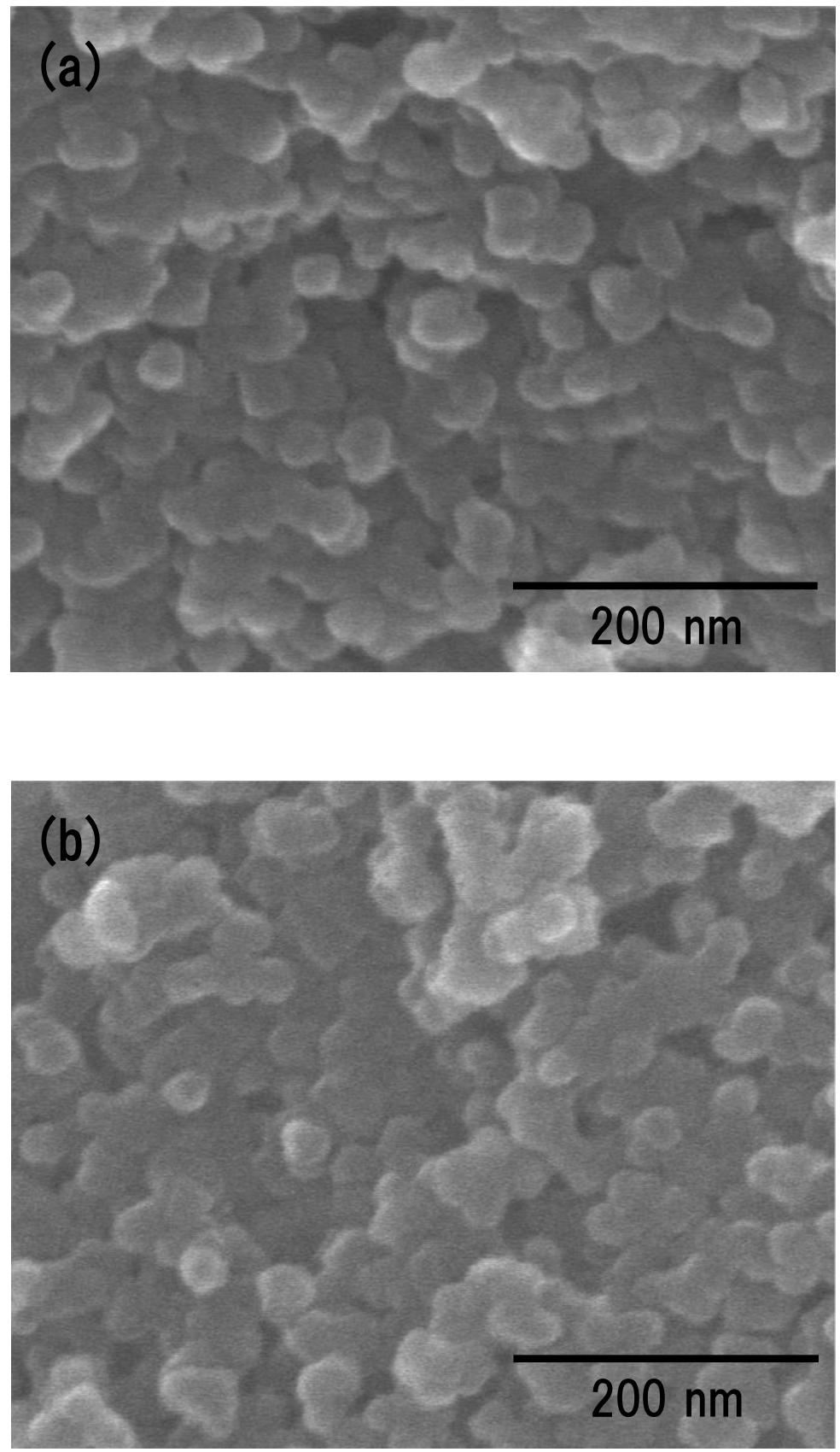

Figure 5 